\title{
Stable Critical Gels of a Crystallizing Copolymer of Ethene and 1-Butene
}

\section{Roland H. Horst*,1 and H. Henning Winter}

Department of Chemical Engineering and Department of Polymer Science \& Engineering, University of Massachusetts, Amherst, Massachusetts 01003

Received J uly 1, 1999; Revised Manuscript Received October 19, 1999

\begin{abstract}
Crystallization of molten polymers involves a liquid-solid transition. In its first stage, the solidification process may be viewed as a physical gelation, which can be monitored with small-amplitude oscillatory shear experiments. A threshold crystallinity, $X_{c g}$, is required for the sample to reach the critical gel state (gel point). Samples of a metallocene random copolymer of ethene and $11 \mathrm{~mol} \%$ 1-butene were quenched from the melt to a crystallization temperature $T_{x}$ and then held at that temperature for isothermal crystallization. The objective was to find the highest value of $T_{x}$ at which the polymer would crystallize only to the threshold crystallinity $\mathrm{X}_{\mathrm{cg}}{ }^{\infty}$ and no further. The result would be a stable critical gel at a temperature $T_{x}=T_{c g}{ }^{\infty}$. We start at large degrees of supercooling where the critical gel is only a transient state which is passed through as the sample solidifies to greater degrees of crystallinity. The gel time increases with temperature, obeying a power law, and can be extrapolated to infinity at $68.8^{\circ} \mathrm{C}$. The crystallinity at the gel point is about $\mathrm{X}_{\mathrm{cg}}=1 \%$, al most independent of $\mathrm{T}$. The rheological experiments are inherently difficult since the characteristic transition behavior, where $\tan \delta$ is independent of frequency, occurs only in the terminal frequency region which is at very low frequencies for the sample of this study.
\end{abstract}

\section{Introduction}

Crystallizing polymers are known to show typical gelation behavior when undergoing a liquid-solid transition (physical gelation). ${ }^{2-4}$ The gel point (GP) in a solidifying system is defined by the appearance of sample spanning connectivity. In chemical gelation, small molecules connect by covalent bonds into larger clusters; the GP is established when the first molecular cluster has grown to infinite size, i.e., to the size of the sample. ${ }^{5}$ Physical gelation results from the growth of long-lived, thermoreversible aggregates which interconnect directly or indirectly. The physical gelation in crystallizing polymers shows many similarities with chemically cross-linking polymers. In the case of the chemical gelation of a two-component system, for instance, the stoichiometric ratio of cross-linker and precursor determines the phase state of the material after completion of reaction. ${ }^{6,7}$ There exist a lower and an upper stoichiometric ratio, $r_{L}$ and $r_{U}$, which define the range of composition in which gelation can occur. Off-balanced reaction mixtures, $r<r_{L}$ or $r_{U}<r$, would not develop sufficient connectivity to establish a sample spanning, three-dimensional molecular network structure. $r_{L}$ and $r_{U}$ are threshold values of composition which, at complete conversion of chemical cross-linking, generate a stable critical gel. The question arises whether a threshold value exists in thermoreversible gelation. Crystallizing conditions are sought which lead to a threshold crystallinity $\mathrm{X}_{\mathrm{cg}}{ }^{\circ}$ for a stable critical gel.

Rheologically, chemical gels and physical gels at the GP express themselves with the same universal relaxation pattern. The critical gel is characterized by a selfsimilar relaxation modulus. ${ }^{8-12}$

\footnotetext{
* Corresponding author. E-mail horst@mail.pse.umass.edu.
}

$$
\mathrm{G}=\mathrm{S}_{\mathrm{c}} \mathrm{t}^{-\mathrm{n}_{\mathrm{c}}} \text { for } \mathrm{t}>\lambda_{0}
$$

$\mathrm{S}_{\mathrm{c}}$ is the gel stiffness, $\mathrm{n}_{\mathrm{c}}$ is the critical relaxation exponent, and $\lambda_{0}$ is the relaxation time denoting the crossover to some faster dynamics (entanglements, segmental dynamics). Therefore, we can use the rheological experiment to detect the gel point in crystallizing samples.

In comparison to polymers, small molecules differ considerably in their crystallization behavior. Crystallization of a one-component, low molar mass system causes a solid, crystalline phase to separate from a liquid, amorphous phase. Only at one specific temperature, the melting point, do both phases coexist; at other temperatures the sample is either totally crystalline or completely amorphous. For polymers, on the other hand, crystallization and melting can occur in a broad temperature range ${ }^{13}$ although polymers can be identified from their transitions determined by DSC or by other means. ${ }^{14}$ At a given crystallization temperature only lamellae of a minimum thickness can form, since the melting point of thinner lamellae would be lower than the crystal lization temperature. The steric constraints of the macromolecules prevent infinite growth of crystals, so the crystall ine fraction never reaches unity. The sample always contains noticeable amounts of amorphous material, and it never reaches the equilibrium melting temperature of the infinite crystal.

Crystallization and melting of polymers are not simple processes; they include several states. ${ }^{15}$ For polyethylene the crystallization process may be viewed as two consecutive main steps: ${ }^{16}(1)$ the formation of lamellae growing radially from a nucleus and (2) the filling of these structures with tangential lamellae. In many cases these structures are spherulites.

For sufficiently high degrees of supercooling, i.e., at a sufficiently low crystallization temperature, the final degree of crystallinity is independent of temperature 
within experimental errors. At higher temperatures it decreases with rising temperature, and above the equilibrium melting temperature, it is zero; i.e., the material is in the melt state. For random copolymers of a crystallizing and a noncrystallizing monomer this curve becomes very flat because of the sequence length distribution. ${ }^{13}$ At a given temperature a minimum thickness of the lamella is required for stability: Uninterrupted sequences of the crystallizable monomers must be longer than this length to be eligible for crystallization. Since the minimum stable lamella thickness increases with temperature, the crystallizable fraction of the copolymer becomes smaller and smaller, causing the flat crystallinity versus temperature curve.

At the gel point, the local structures are interconnected by a supermolecular structure, which is not understood in great detail. But if one considers the possibilities how connectivity can be achieved, three ways come to mind: (i) immediate contact between structural units, (ii) a network of bridging molecules which have segments in neighboring crystalline phases (the tie molecules should be more or less in their equilibrium configuration as long as no external stress is applied. ${ }^{17}$ ), or (iii) impingement of amorphous chains, immobilized by their attachment to segments within a crystalline structure, with si milarly immobilized chains from adjacent structures. Case $\mathrm{i}$ is realized for compounds of low molar mass, whereas polymers seem to pass the macroscopic liquid-solid transition at an earlier stage of structure development. The gel point occurs at so low a crystallinity (ca. 1-2 wt \%) that a solidification according to case $\mathrm{i}$ is most unlikely. The model system for case i would be a suspension of hard spheres solidifying as the volume fraction of the spheres is raised. Solidification of a system of hard spheres by gradual removal of free vol ume between spheres is wellknown. ${ }^{18}$ An experimental example is the suspension of sterically stabilized latex particles. ${ }^{19}$ F or connectivity according to case i the critical gel should be hard and brittle, but semicrystalline polymers of low crystallinity, even beyond the gel point, show a rubberlike behavior, so it is unlikely that they have structure of type (i). Case ii corresponds to network formation during chemical cross-linking, whereas case iii may be compared to a system of swollen spherical microgels in a solvent, the apparent diffusion coefficients of which diverge when the sphere volume reaches the close packing value.20 Crystallizing from the melt state, case iii occurs earlier than case ii since the structures should be closer together to enable bridging. The rheological properties of the critical gel may provide insight into the question of when the gel point is reached. Case ii should result in an elastic rubber, which can only be stretched to a certain amount, whereas case iii should give a sample with a yield stress, which can be elongated infinitely. To date it is unknown exactly at which structural state the macroscopic solidification occurs.

In this study, the terms "solid" and "Iiquid" al ways refer to a macroscopic length scale; the rheometer observes the properties of the overall system, which is solid only after the aforementioned crystal structures are interconnected across the entire sample. This is analogous to Flory's definition of infinite cluster size at the gel point. ${ }^{5} \mathrm{~F}$ or other experiments or considerations, the length scale of interest might be smaller, and then the terms "liquid" and "solid" might be used with a meaning different from the one in this paper. On an intermediate scal e the crystal structures can be considered solid, even if they consist mostly of amorphous polymer, and the phase surrounding the structures can be considered liquid. A microscopic observer, e.g., a small tracer molecule, would only see the crystal phase of the lamellae as solid and everything else as liquid, neglecting the phenomena at the interface. ${ }^{13}$ DSC would also detect a crystal fraction, however, without realizing macroscopic connectivity or lack thereof.

Knowledge of the slightly crystalline state near the gel point is of direct practical importance for polymer processing. An example is vacuum molding which is purposely performed near the gel point where the polymer sheets need to be stiff enough to be selfsupporting in the earlier phase of the process but soft enough to deform under relatively small stress during molding.

For an investigation of this crystallization-induced critical gel state, it is important that the crystallization reaches the gel point at a steady state so that the structure of the polymer at the gel point is stable and can be investigated. The threshold crystallinity $\mathrm{X}_{\mathrm{cg}}{ }^{\circ}$ and the semicrystalline structure will depend on temperature history (path dependence) and other processing parameters (pressure, nucleation aids and other additives, strain). A major difficulty for this experimental study is the critical retardation of the crystallization process near the gel point. In principle, there are two ways to reach a stable critical gel state, either by cooling from the melt state (supercooling) or by heating from the solid state (partial melting). This study focuses on the first possibility, i.e., cooling of a completely molten polymer to a temperature $T_{x}$ which is below its equilibrium melting temperature, $\mathrm{Tm}_{\mathrm{m}}{ }^{0}$, and then observing the changing rheol ogical properties during equilibration at $T_{x}$. We search for the temperature $T_{x}=T_{c g}{ }^{\infty}$ at which the polymer has just enough crystallinity to reach its gel point.

The objective of this paper is to determine whether $\mathrm{T}_{\mathrm{cg}}{ }^{\infty}$ exists and, if so, how it can be approached and what the rheological properties in the stable critical gel state are. It should be much easier to find a temperature where an intermediate degree of crystallinity is realized for a copolymer than for a homopolymer. Early measurements of Flory et al ${ }^{21}$ showed that polymethylene containing randomly distributed ethyl side groups shows a very flat crystallinity versus temperature curve $X(T)$. This is caused by the fact that the branched units are excluded from the crystalline phase. ${ }^{22} \mathrm{~A}$ copolymer of ethylene and 1-butene was chosen for the present measurements, having a much lower melting temperature than pure PE. ${ }^{23}$ DSC will be used to determine the crystallinity as a function of the crystallization conditions. Small-amplitude oscillatory shear experiments will monitor the gelation process, especially the distance of the sample from its gel point.

\section{Experimental Section}

Sample. A statistical copolymer of ethylene and 1-butene, metallocene synthesized, containing 11 mol \% of 1-butene was chosen because of its low crystallinity and its broad melting transition caused by the sequence length distribution. It was used as supplied with a small amount of nucleating agent by Exxon without purification.

Methods. a. DSC. Thermal analysis experiments were performed under nitrogen atmosphere in a Perkin-Elmer calorimeter DSC 7 with water-bath cooling. The instrument was calibrated with high-purity standards of indium and tin. 


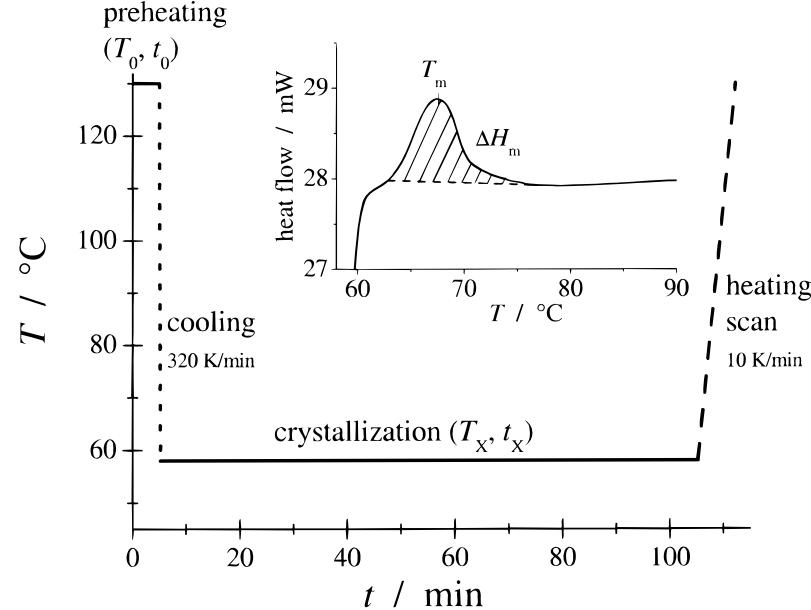

Figure 1. Temperature schedule for the measurements. The sample is preheated at $\mathrm{T}_{0}$ for a certain time $t_{0}$ and then rapidly cool ed to the crystallization temperature $T_{x}$. In the case of the DSC experiments samples are held at $T_{x}$ for some time $t_{x}$. The heat of fusion $\Delta \mathrm{H}_{\mathrm{m}}$ is determined by heating the crystallized sample at $10 \mathrm{~K} / \mathrm{min}$. The oscillatory shear measurements are carried out continuously at $T_{x}$.

b. Rheology. Since stress relaxation experiments require long measuring times, they are not applicable to materials that are changing with time. In this case, the relaxation modulus is better determined in oscillatory shear. Equation 1 can be translated into the frequency domain to give

$$
\begin{gathered}
\mathrm{G}^{\prime}(\omega)=\mathrm{S}_{\mathrm{c}} \Gamma\left(1-\mathrm{n}_{\mathrm{c}}\right) \cos \left(\frac{\mathrm{n}_{\mathrm{c}} \pi}{2}\right) \omega^{\mathrm{n}_{\mathrm{c}}} \text { for } \omega<\omega_{0}=1 / \lambda_{0} \\
\mathrm{G}^{\prime \prime}(\omega)=\mathrm{S}_{\mathrm{C}} \Gamma\left(1-\mathrm{n}_{\mathrm{c}}\right) \sin \left(\frac{\mathrm{n}_{\mathrm{c}} \pi}{2}\right) \omega^{\mathrm{n}_{\mathrm{c}}} \\
\tan \delta=\frac{\mathrm{G}^{\prime \prime}}{\mathrm{G}^{\prime}}=\tan \frac{\mathrm{n}_{\mathrm{C}} \pi}{2} \neq \mathrm{f}(\omega)
\end{gathered}
$$

$\mathrm{G}^{\prime}$ and G" are the storage and loss moduli, $\delta$ is the loss angle, $\omega$ is the angular frequency, and $\Gamma$ is the gamma function. F requency independence of $\delta$ enables the detection of the gel point. Mechanical spectroscopy at a range of frequencies (frequency sweep) yields a constant $\delta$ for a critical gel at sufficiently low frequencies.

Small-amplitude oscillatory shear (SAOS) was performed in a RMS-800 of Rheometric Scientific Inc., equipped with parallel plates (diameter $25 \mathrm{~mm}$ ). The instrument is controlled by Rhios software. After preheating and then cooling to the crystallization temperature $T_{x}$ (about $6 \mathrm{~min}$ ), isothermal SAOS was used to monitor the evolution of loss and storage modulus, G" and G', at a sequence of frequencies between 0.004 and 10 $\mathrm{rad} / \mathrm{s}$. The choice of frequency range was limited by the fact that the time for onefrequency sweep has to be short compared to the gel time; ${ }^{24}$ each data point takes at least one cycle, resulting in very long experimental times for the low frequencies. Therefore, the lowest frequencies could only be used for samples with very long gelation times, i.e., for slow crystallization at high temperatures. The strain amplitude was $\gamma_{a}=$ 0.03 , and in some cases $\gamma_{a}=0.05$. There was no noticeable difference in the results, so strain and shear rate are assumed to be small enough not to influence the crystallization process.

\section{Results}

DSC. In the temperature protocol of Figure 1, the sample was kept at a preheating temperature $T_{0}$ for a certain time $t_{0}$ to remove influences of thermal history as much as possible; then it was quenched at $320 \mathrm{~K} / \mathrm{min}$ to a given crystallization temperature $T_{x}$ and kept there for the time $t_{x}$. In a subsequent DSC heating scan (10
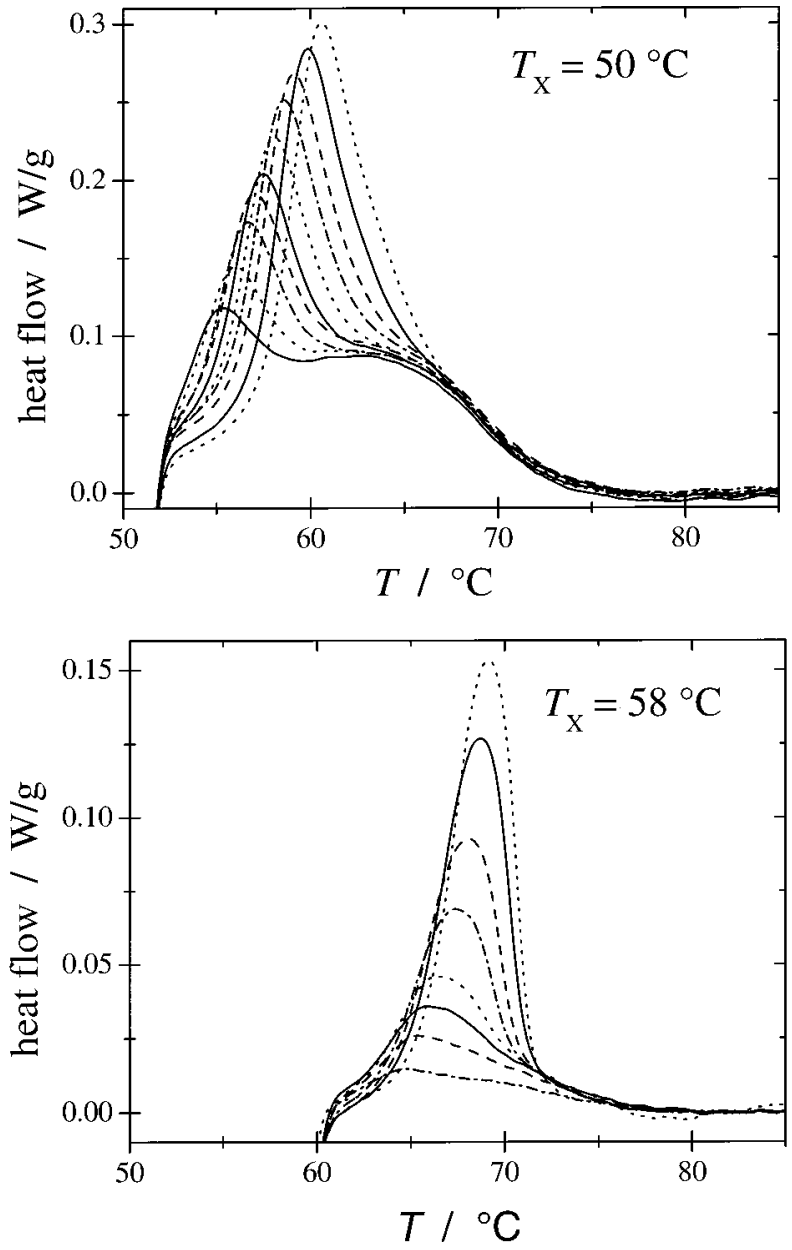

Figure 2. DSC melting curves for constant $T_{x}$ and varying $t_{x}$. The values for $t_{x}$ can be read from Figure 6 , the largest peaks being at $1300,500,200$, and $100 \mathrm{~min}$. The heating rate is $10 \mathrm{~K} / \mathrm{min}$.

$\mathrm{K} / \mathrm{min}$ ), the heat of fusion $\Delta \mathrm{H}_{\mathrm{m}}$ was determined together with the nominal melting temperature $T_{m}$ as defined by the peak maximum.

The actual choice of $T_{0}$ was not obvious, since a plot of $T_{m}$ versus $T_{x}$ did not allow any extrapolation to an equilibrium melting temperature. To overcome this problem, we suggest choosing $T_{0}$ high enough so that the heat of fusion, as measured according to Figure 1 , becomes independent of temperature history. $2^{25} \mathrm{~F}$ or a typical parameter choice $\left(\mathrm{t}_{0}=5 \mathrm{~min}, \mathrm{~T}_{\mathrm{x}}=58^{\circ} \mathrm{C}\right.$, and $t_{x}=30 \mathrm{~min}$ ) in the most sensitive region of our sample, we varied the preheating temperature $\mathrm{T}_{0}$. The heat of fusion $\Delta \mathrm{H}_{\mathrm{m}}$ decreases with increasing $\mathrm{T}_{0}$ in the temperature range below $120{ }^{\circ} \mathrm{C}$; above this temperature $\Delta \mathrm{H}_{\mathrm{m}}$ is constant. On the basis of this observation, the preheating conditions were set at $\mathrm{T}_{0}=130{ }^{\circ} \mathrm{C}$ and $\mathrm{t}_{0}=$ 5 min for all following measurements.

DSC heating scans are shown in Figure 2. The melting process starts almost immediately upon heating; the difference between $T x$ and the peak onset is only $2-5 \mathrm{~K}$, increasing slightly with $t_{x}$. The value of heat flow at the peak maximum and the peak area increase with $t_{x}$ and decrease with $T_{x}$. The peak maximum shifts to higher temperatures with longer annealing times and higher crystallization temperatures. In all cases melting ends at $80^{\circ} \mathrm{C}$. At low $T_{x}$ and short $t_{x}$, a shoulder-or even a second peak-can be seen on the high-temperature side of the DSC-diagram (cf. Figure 2a). 


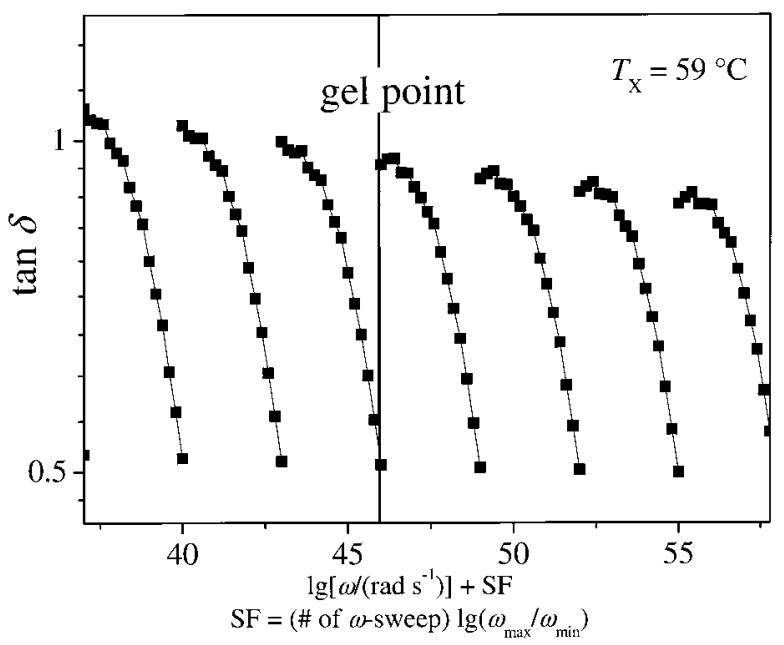

Figure 3. Loss tangent as a function of the angular frequency $\omega$. The curves for the different frequency sweeps are shifted, SF being the shift factor different for each sweep. The gel point is given by a first occurrence of plateau or a maximum at low frequencies, indicated by the vertical line. The shown fre quency sweeps have the numbers 13-19 where (\# of $\omega$-sweep) $=16$ is at the gel point.

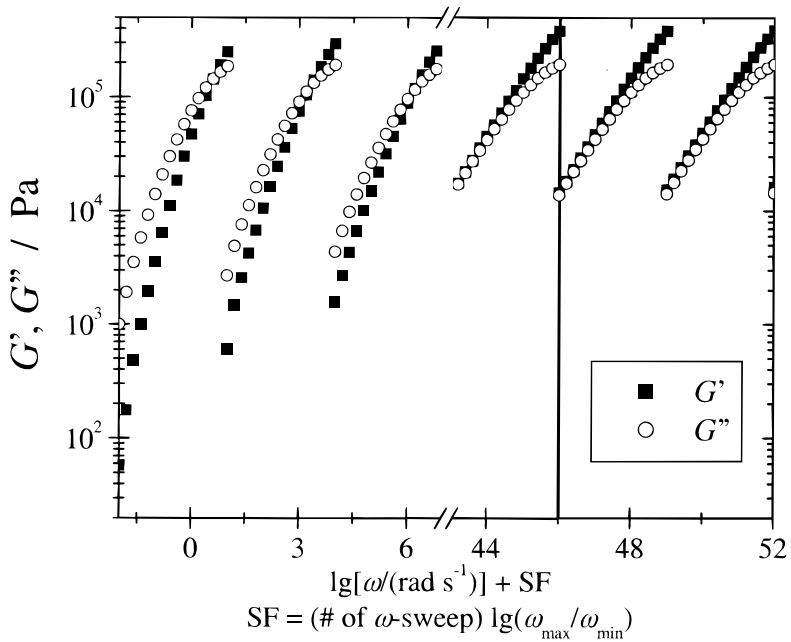

Figure 4. Evolution of the storage ( $\left.\mathrm{G}^{\prime}\right)$ and loss modulus ( $\mathrm{G}^{\prime \prime}$ ). The initial behavior and the behavior at the gel point (vertical solid line) are shown.

Rheology. The temperature schedule for the oscillatory shear experiments was chosen identical to that of the DSC experiments, except for the fact that the cooling from $T_{0}$ to the crystallization temperature $T_{x}$ takes longer, about 6 min. Frequency sweeps were carried out continuously during crystallization at $T_{x}$. Figures 3 and 4 show the evolution of $\tan \delta$ and of the moduli. In a plot of $\tan \delta$ versus $\omega$, the data for the different sweeps overlap. To avoid overlapping in the graphical representation, curves were shifted horizontally by a factor SF so that the curve for one frequency sweep begins where that of the preceding sweep has ended.

\section{Interpretation}

DSC. Multiple melting behavior, 26,27 as expressed in a shoulder or second peak at higher temperatures, also shown in Figure $2 a$, has been reported for various polymers such as isotactic polypropylene (ref 28, Figure $5 a)$, propylene-ethylene copolymers (ref 29, Figure 4b), and poly(butylene terephthalate (ref 30, Figure 2).

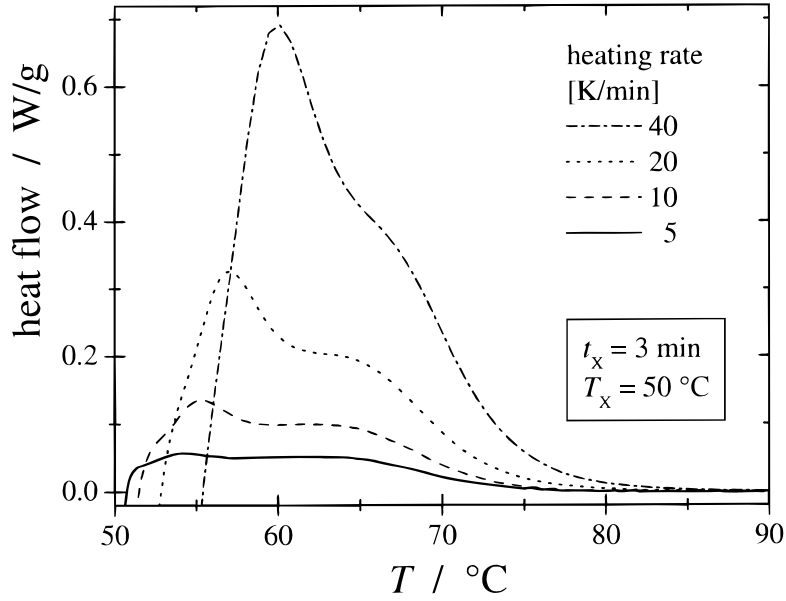

Figure 5. DSC melting curves for different heating rates at constant crystallization conditions of $3 \mathrm{~min}$ at $50{ }^{\circ} \mathrm{C}$.

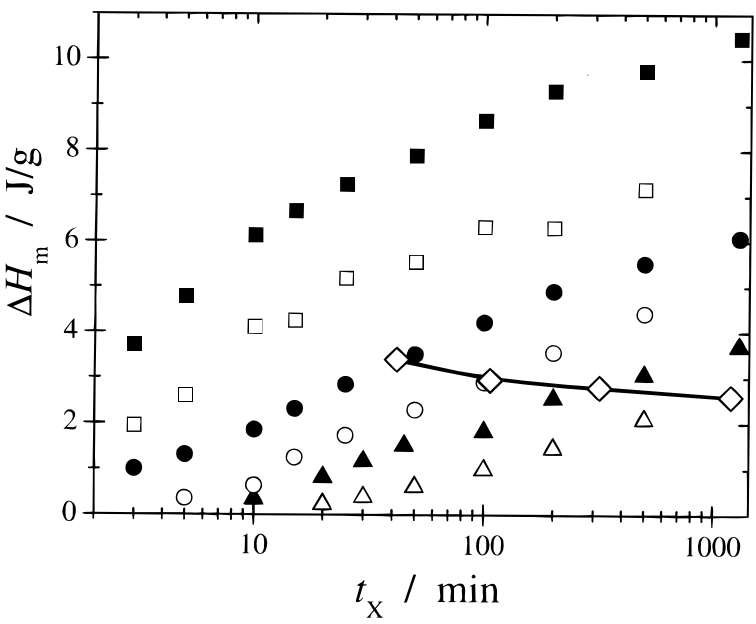

Figure 6. Heat of fusion as a function of the crystallization time for $\mathrm{T}_{\mathrm{X}}=50(\boldsymbol{\square}), 52(\square), 54(\bullet), 56(0), 58(\mathbf{\Delta})$, and $60^{\circ} \mathrm{C}$ $(\triangle)$. The open diamonds $(\diamond)$ and the solid line give the conditions at the gel points.

Multiple melting can be caused by melting of the most unstable crystals and recrystallization at higher temperatures, hence resulting in crystals with a higher melting temperature. The shape of the DSC melting curve is then explained as the superposition of two melting peaks and a negative recrystallization peak in between. An alternative explanation of the double DSC peaks might be polymorphism. However, the second peak or shoulder becomes less pronounced at higher heating rates (see Figure 5), which suggests that recrystallization is its most likely cause. X-ray investigati ons are very difficult for this sample because of its low crystallinity, even at room temperature. One finds a broad peak of the amorphous melt and, superimposed, some very small peaks caused by the crystalline regions. In addition to the peaks of the orthorhombic phase, a peak at lower scattering angle is found which can be attributed to a monoclinic phase. This implies that the cause for multiple peaks could also be polymorphism.

Since it is the goal of this study to find the temperature $T_{x}=T_{c g}{ }^{\infty}$ at which the critical gel state is stable, the experimental temperature was purposely limited to values where the crystallinity remains very small. Unfortunately, this results in very slow crystallization rates. As can be seen in Figure 6, the heat of fusion never reaches a steady value even at $1300 \mathrm{~min}$, roughly 


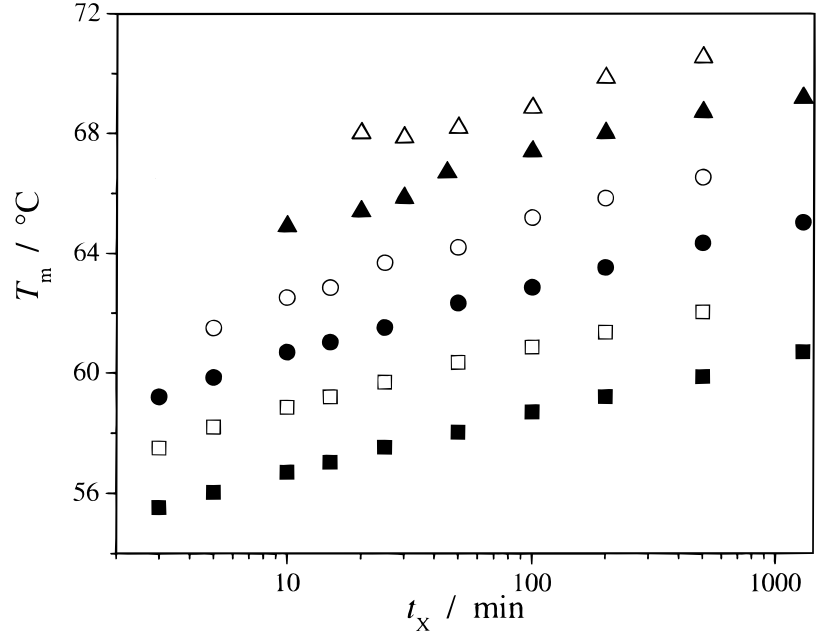

Figure 7. Dependence of the melting temperature $T_{m}$ (position of the maximum of the melting curve) on the crystallization time tx. Symbols as in Figure 6.

1 day. Assuming that no butene units are included in the crystall ine phase, ${ }^{22}$ i.e., the crystalline phase is pure $\mathrm{PE}$, one may assume that the heat of fusion of the crystal is about the same as that for the homopolymer. The value of the polyethylene crystal can be found in the literature $\mathrm{e}^{31}$ and is $295.8 \mathrm{~J} / \mathrm{g}$. Under the above assumptions, dividing the measured heat of fusion by $295.8 \mathrm{~J} / \mathrm{g}$ gives a reasonable value for the crystallinity $X$ of the sample.

In the experimental time and temperature range, no plateau of $\Delta \mathrm{H}_{\mathrm{m}}\left(\mathrm{t}_{\mathrm{x}}\right)$ was reached. The crystallinity increases almost linearly with the logarithm of tx. Only at $50{ }^{\circ} \mathrm{C}$ (see Figure 6) can a slight negative deviation from the straight line be seen. This slowing down of the crystal growth indicates the approach of a plateau.

The "melting" temperature $T_{m}$ at the maximum of the melting peak increases with $t_{x}$ (Figure 7) and $T_{x}$, but it does not reach a plateau value. It increases with $t_{x}$ over the whole time range and runs almost parallel with $T_{x}$ being about $10 \mathrm{~K}$ higher at the long annealing times. This makes it impossible to apply the classical extrapolation to determine the equilibrium melting temperature according to Hoffman-Weeks ${ }^{32}$ or others. ${ }^{33,34}$

Rheology. The frequency independence of $\tan \delta$ gives access to the determination of the gel point. For the case of a crystallizing polymer tan $\delta(\omega)$ should exhibit a negative slope as long as the polymer is in the melt state. At the gel point $\tan \delta$ is independent of $\omega$, at least at low frequencies. Therefore, a plateau should result, as shown schematically in Figure 8. At further solidification the polymer behaves like a solid; the slope of $\tan \delta(\omega)$ is expected to be positive for small $\omega$. The evolution of $\tan \delta$ (Figure 3 ) with time shows that no clearly frequency-independent loss angle was observed. At first the slope is negative in the whole frequency range; later it becomes positive at low frequencies, resulting in a shoulder or a maximum. The reason is that the measurement is not carried out in the terminal zone as can be deduced from Figure 4. The slope of $\mathrm{G}^{\prime}$ is less than two and that of G" less than one. The required low frequencies cannot be realized since than the measuring time would be too long as compared to the gel time. Fortunately, near the threshold temperature, $T_{c g}{ }^{\infty}$, the gel times become very long, and lowfrequency experiments are possible. However, the required low frequencies could not be used since the

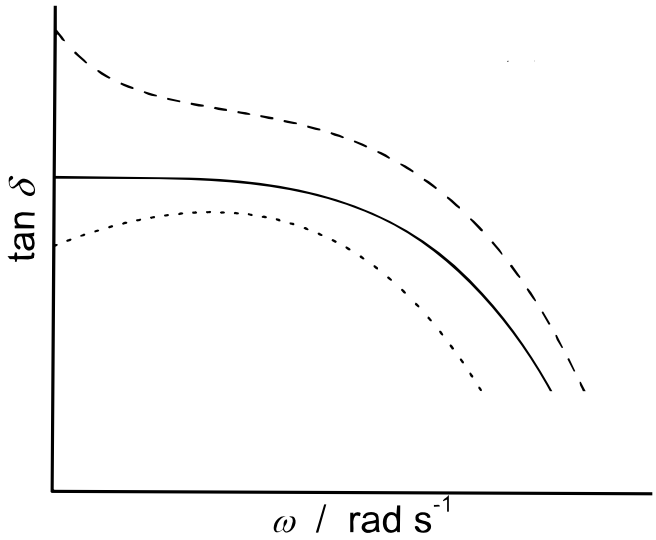

Figure 8. Scheme showing the frequency independence of the loss angle at the gel point (solid line). In the liquid state (dashed line), the slope is negative over the whole frequency range; in the solid it is positive at low $\omega$ (dotted line).

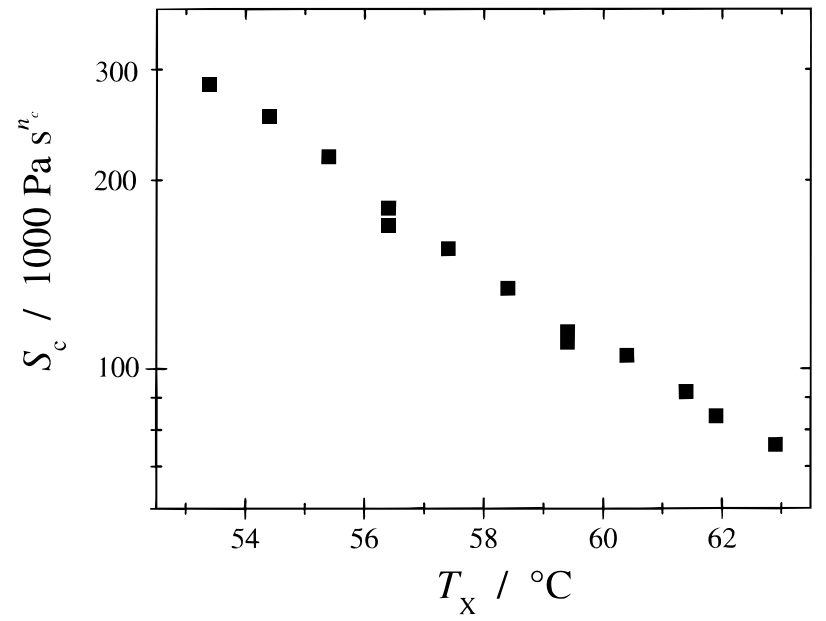

Figure 9. Dependence of the gel stiffness $S_{c}$ on the crystallization temperature.

scattering in the rheological date becomes dominating at frequencies lower than $0.004 \mathrm{~s}^{-1}$, and no reliable determination of the gel point is possible.

Because of these inherent problems, the method to determine the gel time has to be slightly modified. A shoulder or a maximum in $\tan \delta(\omega)$ at low frequencies means that the slope is zero; i.e., $\tan \delta$ is independent of $\omega$, at least at one point. Therefore, the gel point is assumed to have occurred at some point during the first frequency sweep to exhibit a shoulder or a maximum in $\tan \delta(\omega)$. The gel time is estimated as the time when the first data point of this frequency sweep was taken. The error in the gel time is at least the measuring time for one frequency sweep. The estimated gel time might be longer than the actual gel time, since a flat $\tan \delta(\omega)$ might have occurred earlier at frequencies below the experimental range, but the determined value cannot be shorter than the actual gel time.

The two rheological parameters of the critical gels, the gel stiffness $S_{c}$ and the relaxation exponent $n_{c}$ as defined in eqs 2-4, are strongly dependent on $T_{x}$ (Figures 9 and 10). The values for $S_{c}$ and $n_{c}$ were determined at the frequency of the first maximum in $\tan \delta(\omega)$. With increasing $T_{X}$ (less supercooling), the critical gels become softer as expressed in a low $\mathrm{S}_{c}$ value and a high $\mathrm{n}_{\mathrm{c}}$ value. $\mathrm{S}_{\mathrm{c}}$ decreases exponentially with $T_{x}$, and $n_{c}$ increases linearly with $T_{x}$. This is similar for polypropylene. ${ }^{35,36}$ The gel times increase more than 


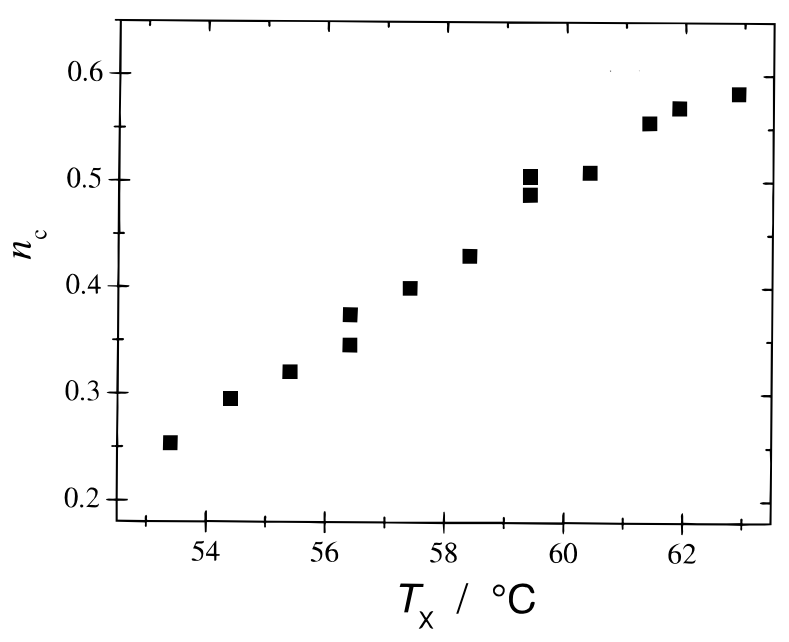

Figure 10. Relaxation exponent $n_{c}$ as a function of $T_{x}$.

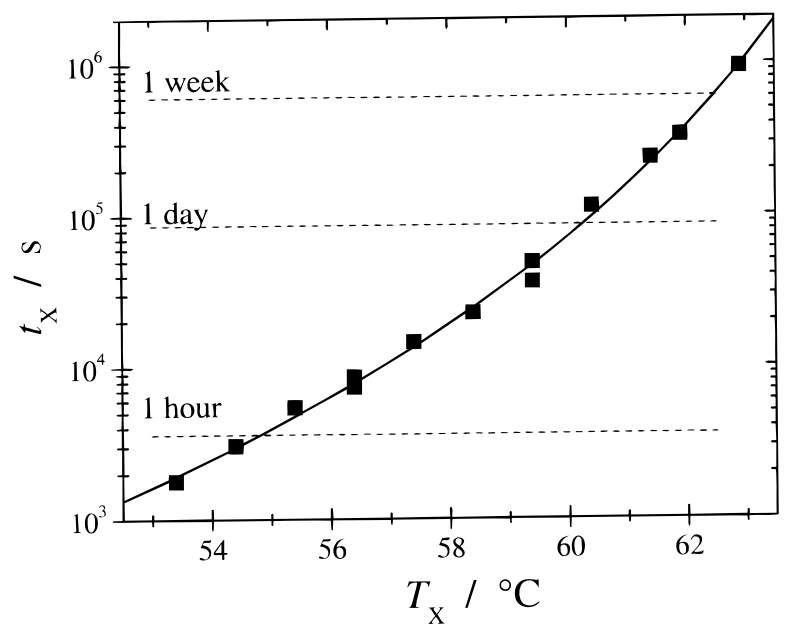

Figure 11. Dependence of the gel time on $T_{x}$. The solid line is the fit according to eq 5 .

exponentially with $T_{x}$ as can be seen in Figure 11.

\section{Discussion}

A power law can describe the dependence of the gel time on the crystallization temperature

$$
t_{g e l}=A\left(\frac{T_{c g}^{\infty}-T_{x}}{T_{c g}^{\infty}}\right)^{-\alpha}
$$

where $\left(T_{c g}{ }^{\infty}-T_{x}\right) / T_{c g}{ }^{\infty}$ is the relative distance from the stable gel point and $\alpha$ is the critical exponent. Fitting of the data gives parameter values $\mathrm{A}=3.87 \times 10^{-6} \mathrm{~s}$, $\mathrm{T}_{\mathrm{cg}}{ }^{\infty}=342.0 \mathrm{~K}$, and $\alpha=6.46$. This function is shown in Figure 11 as solid line together with the experimental points. The validity of eq 5 is better seen in Figure 12 where $t_{g e l}-1 / \alpha$ is shown as a function of $T_{x}$ yielding a straight line.

We define the threshold temperature $T_{\mathrm{cg}}{ }^{\circ}$ by the value of $T_{x}$ at which $t_{g e l}$ diverges to infinity. The intersection with the abscissa at $\mathrm{T}_{\mathrm{cg}}{ }^{\infty}$ marks the point where the crystallization time becomes infinite; i.e., the material requires infinite time to reach the gel point. At these conditions, the solidification reaches the gel point but does not proceed beyond. Furthermore, reaching the gel point at infinite times means the state of the critical gel is stable at $T_{x}=T_{c g}{ }^{\infty}$.

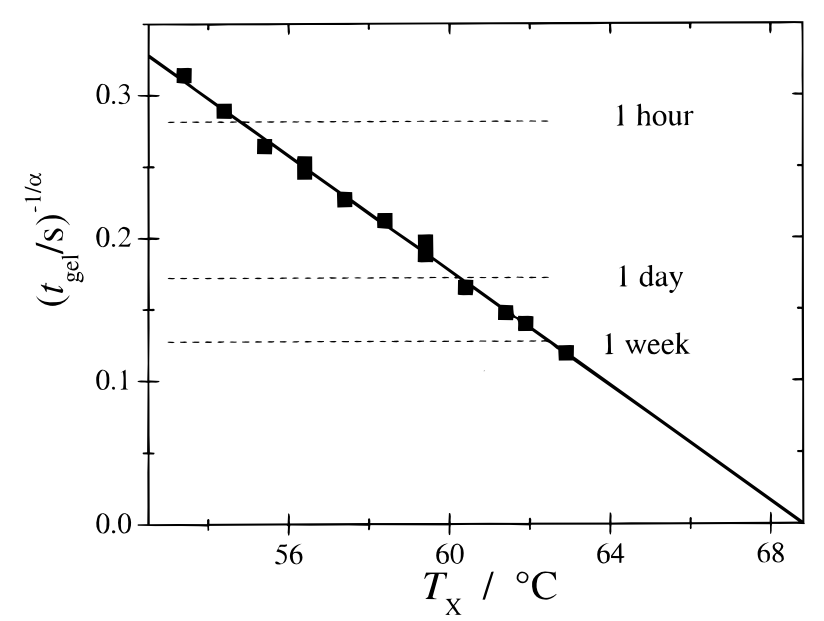

Figure 12. Linearized plot of the gel time versus the crystallization temperature according to eq 5 .

Figure 11 can be read in similar ways as a phase diagram. Below the solid line, indicating the liquidsolid transition, i.e., at high temperatures and short crystallization times, the system is liquid; abovethe line, at low temperatures and long crystallization times, the polymer is solid. At $\mathrm{T}_{\mathrm{cg}}{ }^{\infty}$ the curve diverges; for temperatures higher than $\mathrm{T}_{\mathrm{cg}}{ }^{\infty}$, the polymer is a liquid independent of equilibration time.

It should be noted that $\mathrm{T}_{\mathrm{cg}}{ }^{\infty}$ is not the equilibrium melting point. There is finite crystallinity $\mathrm{X}_{\mathrm{cg}}{ }^{\infty}$ in the polymer, just enough to reach the gel point. The line in Figure 6 gives the heat of fusion at the gel point. It is almost constant at approximately $3 \mathrm{~J} / \mathrm{g}$, which corresponds to $X_{c g}=0.01$, i.e., $1 \%$ crystallinity. This is a much lower value than for polypropylene. ${ }^{35}$ The reason for this very low crystallinity of the critical gel might be the unusual crystallization path. Samples look clear, even at $10 \%$ crystallinity, far beyond the gel point. No large spherulites are formed. The crystalline domains remain very small so that visible light is not markedly scattered. This observation is consistent with the fact that the as-received samples contain a nucleation agent, which promotes growth of many very small crystallites.

\section{Conclusions}

The frequency independence of the loss angle at the gel point can be used to define the material state of the critical gel. The critical gel is a transient state for the crystal lizing polymer. It is reached at longer and longer gel times as the crystallization temperature $T_{x}$ is increased to $T_{\mathrm{cg}}{ }^{\infty}$. The critical gel state, at which the polymer develops sample spanning connectivity for the first time, depends on thermal history; i.e., it is path dependent. However, DSC scans for various critical gels (with $\mathrm{T}_{\mathrm{x}}$ near $\mathrm{T}_{\mathrm{cg}}{ }^{\infty}$ ) are quite similar. The threshold crystallinity is about 1\%, but the melting temperature increases with increasing $T_{x}$ (as expected). At $T_{c g}{ }^{\infty}$ the gel point is reached when the crystallization process is completely finished. Here, the critical gel state is not transient but it is stable. In the case of the crystallizing polymer of this study, a stable critical gel state could not be reached for finite experimental time. The threshold temperature of the stable critical gel was determined by extrapolation to find the temperature $\mathrm{T}_{\mathrm{cg}}{ }^{\infty}$ where $t_{\mathrm{gel}}$ becomes infinite.

The physical gelation caused by crystallization seems very similar to the chemical gelation by cross-linking. 
Crystalline structures connect into a sample spanning network instead of chemical cross-links. Previous studies on the solidification behavior of crystallizing polypropylene ${ }^{35,36}$ showed that the reaction time in the latter case (chemical cross-linking) corresponds to the crystallization time in the former (physical gelation). In this study it was found that the crystal lization temperature correl ates to the stoi chiometric ratio of cross-linker and precursor. Both quantities, $T_{x}$ and the stoichiometric ratio, have a threshold value where the solidification stops exactly at the gel point. For the physical gelation this happens at $\mathrm{T}_{\mathrm{cg}} \mathrm{g}^{\circ}$.

At $\mathrm{T}=\mathrm{T}_{\mathrm{cg}}{ }^{\infty}$, the polymer should exactly reach the threshold crystallinity $\mathrm{X}_{\mathrm{cg}}{ }^{\infty}$ at infinite time; the stable critical gel is realized. At $T<T_{\mathrm{cg}}{ }^{\infty}$, the final crystallinity is higher than $\mathrm{X}_{\mathrm{cg}}{ }^{\infty}$, and the gel time becomes finite; the sample is a solid after sufficient crystallization time ( $t_{x}$ $>t_{\text {gel }}$ ). For $\mathrm{T}>\mathrm{T}_{\mathrm{cg}}{ }^{\infty}$, the crystallinity is too low for a sample spanning connectivity (gel point). If $\mathrm{T}$ is above the equilibrium melting temperature, the sample does not crystallize at all; it is in the melt state.

Furthermore, it should be possible to determine the temperature where the sample behaves as a stable critical gel starting from the solid side by partial melting. The problem here is the much more complex temperature history. The cooling process from the melt, the storage conditions, and finally the heating conditions can influence the solidification behavior. Also, the effect of melting and recrystallization has to be taken into account. Future studies will elucidate this approach from the low-temperature side.

Acknowledgment. The support under the MRSEC program at the University of Massachusetts, Amherst (NSF DMR 9809365), is acknowledged. R.H.H. is grateful to the Deutsche Forschungsgemeinschaft (DFG) for granting a research stipend. We are thankful to Profs. Karasz and MacKnight and their groups for giving access to their DSC equipment.

\section{References and Notes}

(1) On leave from Institute of Physical Chemistry, J ohannes Gutenberg-University, 55099 Mainz, Germany.

(2) te Nijenhuis, K.; Winter, H. H. Macromolecules 1989, 22 $411-414$.

(3) Lin, Y. G.; Mallin, D. T.; Chien, J. C. W.; Winter, H. H. Macromol ecules 1991, 24, 850.

(4) Prasad, A.; Marand, H.; Mandelkern, L. J . Polym. Sci., Polym. Phys. Ed. 1993, 32, 1819-1835.

(5) Flory, P. J . J . Phys. Chem. 1942, 46, 132-140.

(6) Durand, D.; Delsanti, M.; Adam, M.; Luck, J . M. Europhys. Lett. 1987, 3, 297-301.

(7) Winter, H. H. In Encyclopedia of Polymer Science and Engineering, Supplement Volume, Second Edition; Mark, $\mathrm{H}$.
F., Bikales, N. M., Overberger, C. G., Menges, G., Kroschwitz, I. I., Eds.; Wiley: New York, 1989; p 343.

(8) Winter, H. H.; Chambon, F. J . Rheol. 1986, 30, 367-382.

(9) Holly, E. E.; Venkataraman, S. K.; Chambon, F.; Winter, H. H. J. Non-N ewtonian Fluid Mech. 1988, 27, 17-26.

(10) Winter, H. H.; Morganelli, P.; Chambon, F. Macromolecules 1988, 21, 532-535.

(11) Schwittay, C.; Mours, M.; Winter, H. H. Faraday Discuss. 1995, 101, 93-104.

(12) Winter, H. H.; Mours, M. Adv. Polym. Sci. 1997, 134, 165234

(13) Mandelkern, L. In Comprehensive Polymer Science, Vol. 2: Polymer Properties; Allen, G., Bevington, J . C., Eds.; Pergamon Press: Oxford, 1989; p 363.

(14) Menard, K. P. Dynamic Mechanical Analysis: A Practical Introduction; CRC Press: Washington, DC, 1999.

(15) Schmidtke, J .; Strobl, G.; Thurn-Albrecht, T. Macromolecules 1997, 30, 5804-5821.

(16) Akpalu, Y.; Kielhorn, L.; Hsiao, B. S.; Stein, R. S.; Russell, T. P.; van Egmond, J .; Muthukumar, M. Macromolecules 1999, 32, 765-770.

(17) Lustiger, A. In Failure of Plastics; Brostow, W., Corneliussen, R. D., Eds.; Hanser: Munich, 1986; p 314.

(18) Hilbert, D.; Cohn-Vossen, S. Geometry and the Imagination; Chelsea Publishing: New York, 1952.

(19) Weiss, A.; Dingenouts, N.; Ballauff, M.; Senff, H.; Richtering, W. Langmuir 1998, 14, 5083-5087.

(20) Fleischer, G.; Sillescu, H.; Skirda, V. D. Polymer 1994, 35, 1936-1941.

(21) Richardson, M. J .; Flory, P. J .; J ackson, J . B. Polymer 1963 4, 221-236.

(22) Gelfer, M. Y.; Winter, H. H. Macromolecules, in press.

(23) Alamo, R.; Domszy, R.; Mandelkern, L. J . Phys. Chem. 1984, 88, 6587-6595.

(24) Mours, M.; Winter, H. H. Rheol. Acta 1994, 33, 385-397.

(25) Banks, W.; Gordon, M.; Sharples, A. Polymer 1963, 4, 289302.

(26) Petraccone, V.; De Rosa, C.; Guerra, G.; Tuzi, A. Makromol. Chem., Rapid Commun. 1984, 5, 631-634.

(27) Passingham, C.; Hendra, P. J .; Cudby, M. E. A.; Zichy, V.; Weller, M. Eur. Polym. J . 1990, 26, 631-638.

(28) Kamide, K.; Yamaguchi, K. Makromol. Chem. 1972, 162, 205-218.

(29) Feng, Y.; J in, X.; Hay, J . N. Polym. J . 1998, 30, 215-221.

(30) Kim, H. G.; Robertson, R. E. J . Polym. Sci. B, Polym. Phys. 1998, 36, 1757-1767.

(31) Mandelkern, L.; Alamo, R. G. In Physical Properties of Polymers Handbook Mark, E. J .; Ed.; American Institute of Physics: Woodbury, NY, 1996; p 123.

(32) Hoffman, J . D.; Weeks, J. J . J . Res. Natl. Bur. Stand. (U.S.) 1962, A66, 13.

(33) Marand, H.; Xu, J .; Srinivas, S. Macromolecules 1998, 31 $8219-8229$.

(34) Xu, J .; Srinivas, S.; Marand, H.; Agarwal, P. Macromolecules 1998, 31, 8230-8242.

(35) Pogodina, N. V.; Winter, H. H. Macromolecules 1998, 31, 8831-8839.

(36) Pogodina, N. V.; Siddiquee, S. K.; van Egmond, J . W.; Winter, H. H. Macromolecules 1999, 32, 1167-1174.

MA991070X 